\title{
Developmental origins of accelerated cardiovascular aging
}

\author{
Olga Kovtun ${ }^{1}$, and Pavel Tsyvian ${ }^{1,2, *}$ \\ ${ }^{1}$ Ural State Medical University, Department of Physiology, 620028 Repin str.3, Yekaterinburg, \\ Russia \\ ${ }^{2}$ Ural Scientific Research Institute of Maternity and Child Care, Department of Biophysics,620028 \\ Repin str.1, Yekaterinburg, Russia
}

\begin{abstract}
The Barker hypothesis of fetal origins of disease, derived from observational epidemiological studies, has been modified by the realization that the early influences on cardiovascular development may be recognized in the fetus. Aging and developmental programming are both associated with oxidative stress and endothelial dysfunction, suggesting common mechanistic origins. Preeclampsia (PE) is the utmost variant of placental insufficiency and is associated with an increase of serum concentrations of inflammation and antiangiogenic agents in mother and fetus. All these factors are specific for the endothelial dysfunction and able to trigger the epigenetic programming of cardiovascular diseases and accelerated aging. The concentrations of cytokines, tumor necrosis factor, C-reactive protein, NO metabolites,endothelin-1 and homocysteine were measured in serum of women with a normal pregnancy $(n=27), P E(n=30)$ and their newborn infants. The concentrations of all these factors were higher in serum of women and newborns of PE group than in serum of women and newborns of the normal group. The significantly positive correlation between factors concentrations in maternal and newborn's serum was demonstrated in all groups. We suggest the common source of these agents to be the syncytiotrophoblast cells contacting with both maternal and fetal blood and play a significant role in intrauterine programming and epigenetic triggering of accelerated cardiovascular aging.
\end{abstract}

\section{Introduction}

Biomarkers of cellular aging have attracted increasing attention over the past few years. Such biomarkers hold the potential to identify individuals who are at risk of aging-related diseases so that they may be offered timely, targeted preventive interventions, hopefully decades before the onset of disease.

Preeclampsia (PE) is one of the most common complications of pregnancy and is a major cause of maternal, fetal and neonatal morbidity and mortality [1, 2]. PE also leads to increased risk of later hypertension and cardiovascular disease in mother and offspring [36].

\footnotetext{
* Corresponding author: Pavel.tsyvian@gmail.com
} 
To understand the mechanisms that trigger the development of PE, it is important to know how a physiological pregnancy is established and maintained. During normal pregnancy, fetus-derived cytotrophoblast cells migrate through the endometrium and myometrium where they remodel uterine spiral arteries from small diameter, high resistance vessels to larger and more capacitance vessels [7]. Immune cells such as macrophages, uterine natural killers, dendric cells and $\mathrm{T}$ regulatory cells are communicating with cytotrophoblast cells and are required for the normal invasion of cytotrophoblast and vascular remodeling during placentation. Such cellular communication is modulated by the release of cytokines and angiogenic factors. In normal pregnancy, T cells which react with autoantigens are removed by Fas-ligand /Fas-mediated apoptosis, a process known as "clonal deletion" leading to immune tolerance [8]. However, when these reactions, including vasodilation and remodeling are not sufficient, placenta ischemia may occur [8 9]. Several studies have found a significant correlation between reduced utero-placental vascular remodeling and impaired invasion of cytotrophoblast cells into these vessels in PE women [10]. As a result of placental ischemia and subsequent placental inflammation, numerous factors from ischemic placenta such as tumor necrosis factor (TNF $\alpha)$, C-reactive protein (CRP), endothelin-1 and nitric oxide (NO) metabolites release into maternal blood stream. Proinflammatoty cytokines, such as TNF $\alpha$, IL-1 $\beta$, IL-4, IL-10 promote cytotoxic and inflammatory responses [9]. In the vasculature, these agents activate peripheral immune cells mediating targeted endothelial cells, vascular dysfunction and hypertension, a hallmark of PE [11,12].

Homocysteine (Hcy) is formed during the metabolism of dietary methionine. Folic acid and vitamin B12 are required for Hcy metabolism and deficiencies in these can result in increased Hcy concentrations. Hyperhomocysteinemia is a risk factor for both cardiovascular disease and vasculopathy [13].

A striking concept of the etiology of hypertension that has emerged during the last few decades is what is known as developmental programming. This concept refers to the observation that adverse environmental insults early in life, particularly during critical periods of development in-utero and the early postnatal period, may result in long-term morphological and physiological alterations that eventually translate into disease in adulthood $[14,15]$. One can propose that inflammation agents, pro-angiogenic and antiangiogenic factors could affect the fetus and "program" cardiovascular diseases development in later life earlier than usual or trigger premature cardiovascular aging.

The objective of the study is to investigate the possible role in the programming of cardio-metabolic aging and the potential relationship between concentrations of inflammation agents, pro- and anti-angiogenic factors in serum of preeclamptic mothers and their newborn infants.

\section{Material and Methods}

\section{Subjects}

A total of 57 subjects recruited and gave their informed consent at admission to labor and delivery at Maternity and Child Care Research Institute (Yekaterinburg, Russia) as a part of ongoing investigation of preeclampsia and its impact on offspring and approved by the institutional ethics committee. All women gave written informed consent before participation. The research has been carried out in accordance with the Declaration of Helsinki (2013) of the World Medical Association. The design of the project is a crosssectional study. 
Twenty-seven healthy, normal weight $\left(\mathrm{BMI}<25 \mathrm{~kg} / \mathrm{m}^{2}\right)$, normotensive (NT) women with no significant past medical history were included in the control group.

Exclusion criteria were multifetal gestation, chronic hypertension, gestational diabetes mellitus, autoimmune disease, angiopathy, renal disorder, maternal or fetal infection, and fetal congenital anomalies.

Thirty women were included in the preeclampsia (PE) group. Preeclampsia was defined as new onset hypertension that developed after 20 weeks of gestation (systolic or diastolic blood pressure $\geq 140$ and/or $\geq 90 \mathrm{~mm} \mathrm{Hg}$, respectively, measured on at least two occasions, 4 hours to 1 week apart) and proteinuria ( $\geq 300 \mathrm{mg}$ in a 24-hour urine collection, or two random urine specimens obtained 4 hours to 1 week apart containing $\geq 1+$ by dipstick or one dipstick demonstrating $\geq 2+$ protein).

Pregnant women with HELLP syndrome (hemolysis, elevated liver enzymes, and low platelet count) were not enrolled in this study. Also, exclusion criteria for PE group of women were multifetal gestation, diabetes mellitus, autoimmune disease, angiopathy, renal disorder, maternal or fetal infection and fetal congenital anomaly. Fetal intrauterine growth restriction (IUGR) was diagnosed if the fetal birth weight was below the $10^{\text {th }}$ percentile for gestational age and gender. All women were Caucasian and resided in the same geographic area in the Ural region of Russia. All women were non-smokers. Data on pregnancy outcomes were collected from hospital records.

\section{Blood samples}

Blood samples were collected during delivery from the maternal brachial vein after 12-h overnight fasting and from the umbilical vein in tubes containing $1.0 \mathrm{mg} / \mathrm{ml} \mathrm{Na2EDTA.}$ Maternal and cord-blood serum samples were allowed to be kept at room temperature for 1 $\mathrm{h}$, centrifuged at $2000 \mathrm{~g}$ for $20 \mathrm{~min}$, aliquoted under sterile conditions and then stored at $-80^{\circ} \mathrm{C}$ until they were assayed. Serum levels of cytokines (IL-1 $\beta$, IL-4, IL-10, TNF- $\alpha$ ) were measured using system Vector-Best (Vector-Best, Russia). Standard laboratory parameters (clinical chemistry) and C-reactive protein (CRP) levels were determined by an automated analyzer (Biomerica, USA ) using the manufacturer's kits. Endothelin-1 level was assessed by Biomedica system (Biomedica, Austria). Nitrite and nitrate, the stable metabolic products of NO, were measured by nitrate reductase assay using R\&D Systems (R\&D Systems, USA) kit. Plasma total homocysteine concentration was measured using an automated enzymatic assay AxSYM (Abbott, USA).

\section{Statistical Analysis}

The normality of continuous variables was assessed using the Shapiro-Wilk's W-test. As the continuous variables were not normally distributed, nonparametric statistical methods were used. To compare continuous variables among multiple groups, the Kruskal-Wallis analysis of variance by ranks test was performed. Multiple comparisons of mean ranks for all groups were carried out as post-hoc tests. The Fisher exact and Pearson $(\chi 2)$ tests were used to compare categorical variables between groups. The Spearman rank order correlation was applied to calculate continuous variables. Statistical analyses were performed using STATISTICA package (version 8.0; StatSoft Inc., USA). In the case of the subordination of the distribution of a trait to the law of normal distribution, the data were presented as a mean \pm SE. In the case of a deviation from the normal distribution, data are reported as median (25th and 75th percentiles, LQ and UQ) for continuous variables and as number (percentage) for categorical variables. In order to overcome the problem of multiple 
comparisons, the Bonferroni correction was applied. The critical significance level of the differences ( $\mathrm{p}$ ) at which the null hypothesis about the absence of differences was rejected and the alternative hypothesis was adopted, was set at equal to 0.017 .

\section{Results and Discussion}

Characteristics of the study population are shown in Table 1. There was no statistically significant difference in terms of age among women of the study groups.

Table 1. Maternal and newborn characteristics.

\begin{tabular}{lcc}
\hline Maternal characteristics & $\begin{array}{c}\text { PE } \\
(\mathrm{n}=30)\end{array}$ & $\begin{array}{c}\text { Uncomplicated } \\
\text { controls (n=27) }\end{array}$ \\
\hline Age (years) & $30(28-34)$ & $27(25-34)$ \\
Mean AP $(\mathrm{mm} \mathrm{Hg})$ & $118.9 \pm 1.3^{*}$ & $88.1 \pm 1.1$ \\
Preconception BMI $\left(\mathrm{kg} / \mathrm{m}^{2}\right)$ & $26.8 \pm 0.5^{*}$ & $24.7 \pm 0.6$ \\
Nulliparous n (\%) & $24(80 \%)^{*}$ & $9(33 \%)$ \\
Caesarean section n (\%) & $25(83 \%)^{*}$ & $4(15 \%)$ \\
\hline Newborn characteristics & & \\
Gestational age at birth (weeks) & $31.0(29.5-33.0)^{*}$ & $39.0(39.0-40.0)$ \\
Birth weight (grams) & $1160 \pm 125^{*}$ & $3417 \pm 91$ \\
Birth weight $<10$ percentile n (\%) & $17(56.4 \%)^{*}$ & 0 \\
\hline
\end{tabular}

Data are presented as Mean \pm SE.

Non-parametric data are presented as median (interquartile range) with corresponding KruskallWallis testing.

$\mathrm{AP}$-arterial pressure

BMI - body mass index

$*$ - $\mathrm{p}<0.01-$ The significance of the difference between study and control groups;

Mean blood pressure was significantly higher $(\mathrm{p}<0.001)$ in PE group in comparison to the control group. All PE women received the hypertension treatment before the delivery. Women with PE delivered at an earlier gestational age and their babies were significantly smaller than in normotensive women. These differences were statistically significant.

Fetal growth restriction was absent in the normotensive subset, but occurred in $17 \%$ $(\mathrm{p}<0.001)$ of the PE group.

The laboratory parameters of the study subjects are presented in Tables 2 and 3.

Table 2. The concentrations of proinflammatory mediators and markers of endothelial dysfunction in mothers of investigated groups

\begin{tabular}{lcc}
\hline \multicolumn{1}{c}{ Parameters } & $\begin{array}{c}\text { PE } \\
(\mathrm{n}=30)\end{array}$ & $\begin{array}{c}\text { Uncomplicated controls } \\
(\mathrm{n}=27)\end{array}$ \\
\hline Endothelin-1, fmol/ml & $0.55(0.3-1.25)^{*}$ & $0.09(0.01-0.27)$ \\
$\mathrm{Total}^{*} \mathrm{NO}_{2}^{-}, \mu \mathrm{mol} / \mathrm{L}$ & $13.71(10.26-15.37)^{*}$ & $9.75(8.73-11.28)$ \\
$\mathrm{NO}_{3}{ }^{*}, \mu \mathrm{mol} / \mathrm{L}$ & $11.76(8.78-12.99)^{*}$ & $8.51(7.6-9.42)$ \\
$\mathrm{Homocysteine}^{*} \mu \mathrm{mol} / \mathrm{L}$ & $15.08(10.37-18.95)^{*}$ & $7.5(2.83-10.06)$ \\
$\mathrm{IL}-1 \beta, \mathrm{pg} / \mathrm{ml}$ & $1.36(0.78-2.7)^{*}$ & $1.07(0.86-1.96)$ \\
$\mathrm{IL}-4, \mathrm{pg} / \mathrm{ml}$ & $5.68(1.41-9.73)^{*}$ & $1.54(1.46-2.85)$ \\
$\mathrm{IL}-10, \mathrm{pg} / \mathrm{ml}$ & $19,85(6,17-21,68)^{*}$ & $4,37(4,03-4,55)$ \\
$\mathrm{TNF} \alpha, \mathrm{pg} / \mathrm{ml}$ & $50,54(26,0-60,8)^{*}$ & $23,1(15,67-30,2)$ \\
C-reactive protein, $\mathrm{mg} / \mathrm{L}$ & $9,3(8,9-10,0)^{*}$ & $4,8(4.28-6.73)$ \\
\hline
\end{tabular}

$* \mathrm{p}<0.017-$ The significance of the difference between PE and control groups 
Table 3. The concentrations of proinflammatory mediators and markers of endothelial dysfunction in newborn infants of investigated groups

\begin{tabular}{lcc}
\hline \multicolumn{1}{c}{ Parameters } & $\begin{array}{c}\text { PE } \\
(\mathrm{n}=30)\end{array}$ & $\begin{array}{c}\text { Uncomplicated controls } \\
(\mathrm{n}=27)\end{array}$ \\
\hline Endothelin-1, fmol/ml & $0.53(0.36-0.58)^{*}$ & $0.09(0.07-0.3)$ \\
Total $\mathrm{NO}_{2}{ }^{-}, \mu \mathrm{mol} / \mathrm{L}$ & $10.41(7.33-13.07)^{*}$ & $6.81(5.56-10.08)$ \\
$\mathrm{NO}_{3}{ }^{*}, \mu \mathrm{mol} / \mathrm{L}$ & $8.29(5.44-11.94)^{*}$ & $6.84(6.1-10.3)$ \\
$\mathrm{Homocysteine}, \mu \mathrm{mol} / \mathrm{L}$ & $10.4(8.23-12.91)^{*}$ & $6.81(2.47-7.54)$ \\
$\mathrm{IL}-1 \beta, \mathrm{pg} / \mathrm{ml}$ & $2.28(0.99-5.37)^{*}$ & $0.86(0.67-1.14)$ \\
$\mathrm{IL}-4, \mathrm{pg} / \mathrm{ml}$ & $3.77(1.62-5.84)^{*}$ & $1.55(1.48-4.85)$ \\
$\mathrm{IL}-10, \mathrm{pg} / \mathrm{ml}$ & $6.48(3.64-10.08)^{*}$ & $4,06(3.82-4,92)$ \\
$\mathrm{TNF} \alpha, \mathrm{pg} / \mathrm{ml}$ & $35.73(20.07-49.92)^{*}$ & $21.41(13.9-25.2)$ \\
C-reactive protein, $\mathrm{mg} / \mathrm{L}$ & $0.25(0.18-0.35)^{*}$ & $0.1(0.05-0.15)$ \\
\hline
\end{tabular}

$* \mathrm{p}<0.017-$ The significance of the difference between PE and control groups

Women who developed PE demonstrated higher $(\mathrm{p}<0.017)$ concentrations of the main markers of endothelial dysfunction and inflammation compared to the normotensive women. The concentrations of stable NO metabolites, cytokines ( IL-1 $\beta$, Il-4, IL-10, TNF $\alpha$ ) and C-reactive protein were significantly higher in serum of PE women than the normotensive subset. Total $\mathrm{NO}^{-}$concentration in serum of PE women was 5.9 times higher $(\mathrm{p}<0.017)$ than in than in women of control group.

Endothelin-1 and homocysteine concentrations in serum of PE women were higher $(\mathrm{p}<$ 0.017 ) than in normotensive women (Table 2). A similar pattern was demonstrated in serum of newborn infants $(\mathrm{p}<0.017)$. NO metabolites and main inflammation markers were significantly higher $(\mathrm{p}<0.017)$ in the serum of PE infants. PE infants demonstrated Endothelin-1 concentration more than 5 times higher $(p<0.017)$ than control infants. Homocysteine and CRP concentrations were significantly higher $(\mathrm{p}<0.017)$ in serum of PE infants than in the control group (Table 3).

A high correlation between concentrations of all factors in the serum of mothers and their infants was demonstrated in PE group and in the control group (Table 4).

Table 4. Spearman rank order correlation coefficients between investigated analytes in maternal plasma and newborns cord blood of PE and control groups.

\begin{tabular}{|c|c|c|c|c|}
\hline \multirow[t]{2}{*}{ Parameters } & \multicolumn{2}{|c|}{$\begin{array}{c}\mathrm{PE} \\
(\mathrm{n}=30)\end{array}$} & \multicolumn{2}{|c|}{$\begin{array}{l}\text { Uncomplicated } \\
\text { controls } \\
(\mathrm{n}=27)\end{array}$} \\
\hline & $\mathrm{R}$ & $\mathrm{P}$ & $\mathrm{R}$ & $\mathrm{P}$ \\
\hline Endothelin-1, fmol/ml & 0.58 & 0.0001 & 0.45 & 0.01 \\
\hline Total $\mathrm{NO}_{2}^{-}, \mu \mathrm{mol} / \mathrm{L}$ & 0.41 & 0.001 & 0.47 & 0.01 \\
\hline $\mathrm{NO}_{3}{ }^{-}, \mu \mathrm{mol} / \mathrm{L}$ & 0.69 & 0.0001 & 0.53 & 0.0001 \\
\hline Homocysteine, $\mu \mathrm{mol} / \mathrm{L}$ & 0.71 & 0.0001 & 0.79 & 0.0001 \\
\hline $\mathrm{IL}-1 \beta, \mathrm{pg} / \mathrm{ml}$ & 0.55 & 0.001 & 0.66 & 0.001 \\
\hline IL-4, pg/ml & 0.45 & 0.001 & 0.71 & 0.0001 \\
\hline $\mathrm{IL}-10, \mathrm{pg} / \mathrm{ml}$ & 0.48 & 0.001 & 0.73 & 0.0001 \\
\hline $\mathrm{TNF} \alpha, \mathrm{pg} / \mathrm{ml}$ & 0.75 & 0.0001 & 0.70 & 0.0001 \\
\hline C-reactive protein, $\mathrm{mg} / \mathrm{L}$ & 0.36 & 0.01 & 0.23 & 0.05 \\
\hline
\end{tabular}

$\mathrm{R}$ - Spearman rank order correlation coefficient

$\mathrm{p}$ - Significance 
Numerous studies have demonstrated important steps in the progression of preeclampsia from placental underperfusion and hypoxia to a clinical syndrome among which an increase in levels of circulating antiangiogenic factors, activation of inflammation and immunological imbalance [1,6]. In this study, a significant increase in vasoconstrictor factor endothelin-1 was demonstrated in preeclampsia compared to normotensive women. This is consistent with previous studies [16].

One of the main markers of endothelial dysfunction - hyperhomocysteinemia - was shown in the PE group. This is in line with previously demonstrated results that Hcy is increased in PE and eclampsia [17].

It has been assumed that higher levels of Hcy may contribute to the development of placental microvascular disease and preeclampsia (PE), thus adversely affecting the endothelium [18]. Vascular damage in PE groups of mothers and offspring is also confirmed by the increased concentration of CRP which is nonspecific indicator of inflammation [17]. The immunologic component of PE is reflected by increased TNF- $\alpha$, Il1 and IL-4 in groups of mothers and infants. The maternal systemic inflammatory response characteristic in preeclampsia involves an acute-phase reaction as well as oxidative stress, and circulating cytokines could play a pivotal role in these processes [19]. Cytokines can cause the release of oxygen free radicals, which in turn may enhance the endothelial damage. The role of cytokines in PE pathogenesis was assessed in several studies [20, 21]. This is consistent with previous study examining placental microvesicles from preeclamptic women, demonstrating significantly increased secretion of IL-1 $\beta$ compared to microvesicles from normal term placentas [22]. In our study, circulating levels of the proinflammatory cytokines IL- $1 \beta, \mathrm{TNF}-\alpha$ as well as $\mathrm{C}$ reactive protein were raised in PE women compared to normotensive women, resulting in an overall pro-inflammatory systemic environment.

In this study we simultaneously measured inflammatory and endothelial markers both in maternal and offspring serum. It is of interest that all analytes increased in maternal plasma PE group were also increased in serum of their newborn infants.

One of the most fascinating properties of the normally developing pregnancy is the delicate interaction between fetal-derived trophoblast cells and maternal cells. This interaction or fetal-maternal dialog is synchronized by the production of cytokines, growth factors, NO metabolites and hormones to establish an unique maternal-fetal immune tolerance that is essential for the spiral artery remodeling, fetal survival and development $[23,24]$. Dysfunction in this interaction of trophoblast cells to the mother-derived cells is linked with some pregnancy failures, such as miscarriage, preeclampsia and fetal growth restriction [25].

Studies in young adults demonstrated that fetal growth restriction is associated with impaired arterial endothelial function [26]. We found evidence for elevated levels of the inflammatory markers (CRP, cytokines) and markers of endothelial dysfunction (Endothelin-1) in the serum of pre-eclamptic mothers and their offspring. Maternal preeclampsia and gestational hypertension have been associated with a higher blood pressure in offspring during childhood and adolescence. Increased systolic blood pressure in early adolescence was demonstrated in offspring of preeclamptic women [27]. Preeclampsia and gestational hypertension were both associated with increased systolic and diastolic blood pressure in nine-year-old offspring [28].

The underlying mechanisms explaining the associations of gestational hypertension and preeclampsia with offspring blood pressure remain unclear. A striking concept on the etiology of hypertension that has emerged during the last few decades is what is known as developmental programming or Developmental Origins of Health and Disease (DOHaD) hypothesis [29]. This concept refers to the observation that adverse environmental insults early in life, particularly during critical periods of development in utero and the early 
postnatal period, can result in long-term morphological and physiological alterations that eventually translate into disease in adulthood [30]. Biomarkers of such intrauterine programming have attracted increasing attention. One of these biomarkers is DNA methylation which is epigenetic mechanism characterized by addition of one methyl group primarily to cytosine-phosphate-guanine $(\mathrm{CpG})$ sites of DNA [3]. Significant increase in DNA methylation was demonstrated in offspring of PE mothers [3]. This process was also demonstrated during aging [3]

\section{Conclusion}

We demonstrated a significant correlation between angiogentic factors, cytokine concentrations and NO metabolites in serum of mothers and newborn infants of all groups. This phenomenon could be explained by the possible transplacental transport of these factors. On the other hand one could suggest the common source of these agents could be the syncytiotrophoblast cells contacting with both maternal and fetal blood [18]. Thus the placenta playing a leading role in the establishment of a unique fetal-maternal "dialog", endothelial function and intrauterine programming of cardiovascular aging in later life.

\section{References}

1. B.M. Sibai, Obstet. Gynecol., 102, 181 (2003)

2. L.C.Chappell, S. Enye, P.Seed P, Hypertens., 51, 1002 (2008)

3. E.V. Herzog, A.J. Eggink, A. Reijnierse, Placenta., 49, 72 (2017)

4. E. Kajantie, J.G. Eriksson, C. Osmond, K. Thornburg, Stroke, 40, 1176 (2009)

5. Tranquilli, M. Brown, Z. Zeeman, Pregn. Hypertens. An Int. J. Women's Cardiovasc. Health, 44, e47 (2013)

6. D. Raymond, E. Peterson, Obstet. Gynecol. Surv., 8, 497 (2011)

7. G.S. Whitley, J.E. Cartwright, Placenta.31, 465 (2010)

8. S.A. Saito, A. Nakashima, M.Sakai, Y.Sasaki, Mol. Asp. Med., 28, 192 (2007)

9. J.M. Roberts, Semin. Perinatol., 38, 139 (2014)

10. F. Lyall, S.C. Robson, J.N.Bulmer, Hypertens., 62, 1046 (2013)

11. Kharfi, Y. Giguere, V. Sapin, Clin. Biochem., 36, 323 (2003)

12. B.B. La Marca, D. Cornelius, K. Wallace, Physiology, 28, 225 (2013)

13. G.N. Welch, J. Loscalzo, N. Engl. J. Med., 338, 1042 (1998)

14. A.M. Cotter, A.M. Molloy, J.M. Scott, S.F. Daly, Am. J. Obstet. Gynecol., 189, 391 (2003)

15. K. Zandi-Nejad, V.A. Luyckx, B.M. Brenner, Hypertens., 47, 502 (2006)

16. J.S.Gilbert, M.J. Ryan, B.B. La Marca B B, Amer. J. Physiol. Heart. Circ.Physiol., 294, H541 (2008)

17. M. Ingec, B. Borekci, S. Kadanali, Tohoku J. Exp.Med., 206, 225 (2005)

18. F. Sun, W. Qian, C. Zhang, Med. Sci. Monit.,23, 5396 (2017)

19. S. Maynard, F.H. Epstein, S.A. Karumanchi, Annu. Rev. Med.,59, 61 (2008)

20. B.D.Taylor, G.Tang, R.B. Ness, Pregn. Hypertens., 6, 72 (2016)

21. M. Kalinderis, A. Papanikolaou, K. Kalinderi, Am. J. Reprod. Immunol., 66, 468 (2011)

22. B.S. Holder, C.L. Tower, C.J. Jones, J.D. Aplin, Biol. Reprod., 86, 103 (2012)

23. A.L.Veenstra van Nieuwenhoven, M.J. Heineman, M.M. Faas, Hum. Reprod. Update, 9, 347 (2003)

24. E. Dimitriadis, C.A.White,R.L. Jones, L.A. Salamonsen, Hum. Reprod. Update., 11, $613(2005)$

25. E. Ball, J.N.Bulmer, S. Ayis S, J. Pathol., 208, 535 (2006) 
26. C.P. Leeson, M. Kattenhorn, R. Morley, Circulation., 103, 1264 (2001)

27. B. Oglaend, M.R. Forman, P.R. Romundstad, J. Hypertens., 27, 2051 (2009)

28. J.J. Geelhoed, A. Fraser, K. Tilling, Circulation, 122, 1192 (2010)

29. D.J. Barker, J.Intern. Med., 261, 412 (2007)

30. P.D. Gluckman, M.A. Hanson, Pediatr . Res., 56, 311 (2004) 